\title{
Real-time Animation of Bird Flight Based on Aerodynamics
}

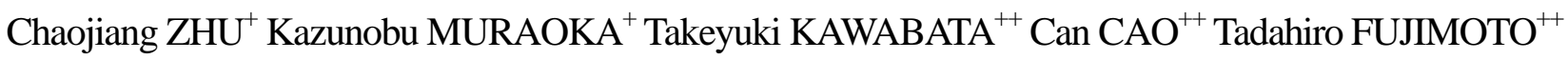 \\ and Norishige CHIBA ${ }^{++}$ \\ ${ }^{+}$Tohoku Institute of Technology ${ }^{++}$Iwate University
}

\begin{abstract}
The expression of animals and natural phenomena as simulated by computer graphics can be expected to enhance the realism of landscape simulation, virtual reality, etc. In this paper, a model of bird flight model animated in real time is proposed by taking aerodynamics into consideration. In this model, a bird flies by the wingbeat motion and using its tail feathers. Furthermore, target points are set in the space and the bird's orientation and flapping are adjusted such that the bird passes through these target points in sequence. This makes it possible to have the bird fly along an arbitrary path.
\end{abstract}

Key word: bird, real-time visual simulation, animation, aerodynamics

\section{Introduction}

Computer graphics (CG) animation of animals is an important aspect for increasing realism in applications such as landscape simulation and virtual reality (VR) and for conveying seasonal environmental cues. Birds can be found in almost all parts of the world, and many species migrate. Their migratory behavior is immediately recognizable as the feature of a particular season, and in fact, birds are one of the most suitable fauna for indicating a season; thus, they are gaining increasing importance in computer graphics.

Previous CG simulations of bird models were primarily of two types: the rule-based simulation, which consists of an automatic generation of bird motions according to certain rules, and the flapping-driven simulation, in which the force calculated by the wingbeat motion is adopted as the governing principle for bird motions. As an example of the former model (rule-based simulation), Reynolds [1] simulated the flight of a flock of birds in a highly realistic manner. However, in this method, the wingbeat motion has no physical meaning, and while the flight may appear realistic at a distance, there is a possibility that the overall motion will appear unnatural. In the flapping-driven simulation, there is a cause-and-effect relationship between the wingbeats and the bird's flight, and hence, natural flapping can be simulated. Ramakrishnananda et al. [2] proposed a model for the flight of large birds in which the bird is propelled by the force of wingbeats and moves up and down toward its target points. It has been shown that it is possible to express the motion of the bird naturally by using the force of wingbeats to move the bird. However, the calculation time of their method was longer than the true bird-flight time, precluding real-time display. In addition, the bird is restricted to only forward movement; it cannot perform a circular flight. Wu et al. [3] proposed a realistic model of bird flight. Their model allows a circular flight, but an optimization process is required as flight pre-preparation; moreover, the birds do not move in real time. On the other hand, Cao et al. [6] applied the flapping-driven model by assuming a quasi-steady state air flow for a butterfly, as proposed by Anzai et al. [7], to a bird and enabled the bird to fly in real time. However, since the circular flight of the bird is performed at a fixed rotation speed, its movement may appear unnatural. Furthermore, in their model, the takeoff and landing motions are not taken into consideration.

In this study, the influence of the force produced by the tail feathers is added to Cao's bird flight model, and a method is proposed that can realize a realistic circular flight. Further, methods are proposed to simulate bird flight taking takeoff into consideration, the ground effect when a bird flies close to the surface of the ground and flight path control when the bird lands on a branch [9].

The purpose of this study is to apply to real-time applications, such as a driving simulator and virtual reality. For this reason, in our technique, the geometric model and the calculation model are simplified much more than the method of no-real time, for example, the model of Wu [3]. Simplification is unavoidable in order to perform real-time operation in common PC. In the model of Wu, each of the wing feathers is modeled and aerodynamic forces acting on them are calculated respectively. Compared to it, in our model, we only calculate five points, including two points for each wing and one point for tail feathers. Moreover, our model's joints are few then the Wu's model. However, in the real world, it is rare for people to observe wild birds nearby, and in many cases we often keep dozens of meters away. In this distance, although the form of each feather and its motions can hardly be recognized, a movement of wings and motions of tail feathers are easily distinguished. The model proposed by us is enough to express the operation of wing and tail feathers. Moreover, it is possible to change flight course freely during flight. According to this feature, it can be made to simulate that birds avoid moving cars or people in a driving simulator and virtual reality.

It shall be noted that these methods are strictly techniques to pursue the realism of the appearance of the flight of a bird for visual simulation; they do not analyze the power produced by the wingbeats of a bird.

\section{Actual Bird Flight Features}

Based on the literature $[4,5]$, a summary of the actual form features of a bird and its flight actions is presented 
below.

\subsection{Form Features of Bird}

A bird's wing is similar to the human arm in that it consists of three parts: the upper arm, forearm, and hand, along with three joints: the shoulder, elbow, and wrist. The feathers that cover the wing are categorized as coverts, primary flight feathers, and secondary flight feathers (see Fig. 1).

\subsection{Flight Motion of Bird}

There are three fundamental motions of the wing movement: flapping, feathering, and lead-lag motions. The flapping motion comprises the upward and downward movements of the entire wing around the shoulder. The feathering motion comprises the upward and downward movements of the flight feathers with the leading edge of the wing as the rotation axis. The lead-lag motion comprises the forward and backward movements of the wing tip (see Fig. 2).

The tail feathers are instrumental in stabilizing the flight, changing the direction of the forward movement, compensating for the lift force, and acting as a brake when the bird lands. As shown in Fig. 3(a), when a bird rotates, the tail feathers are twisted either to the right or left. Moreover, in order to compensate for the lift force, the tail feathers are adjusted by bending them upward or downward during a rise (see Fig. 3(b)). Moreover, the force produced by the tail feathers is altered by adjusting the opening-and-closing angle and changing the area of the tail feathers (see Fig. 3(c)). The tail feathers open when a bird flies toward the right or left or when a lift force is required, and they close during cruising flight so that the drag force is reduced.

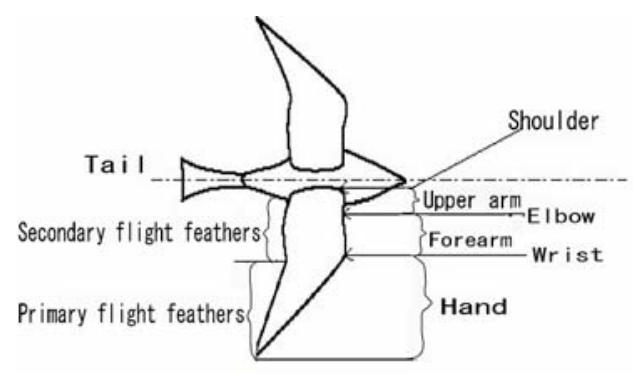

Fig. 1 Structure of bird wings

\section{Foundation of Bird Flight Model}

Cao's bird flight model is described briefly in this section.

\subsection{Bird Model for Wingbeat Calculation}

In order to calculate the wingbeat force, a bird is assumed to be flying in a quasi-steady state air flow and is modeled as shown in Fig. 4. In this model, the wing is represented by an arm and hand that rotate around the shoulder and wrist. Further, the wings perform flapping, feathering, and lead-lag motions [8].

\subsection{Wingbeat Movement}

In order to realize the three types of wingbeat movement (flapping, feathering, and lead-lag), an angle function was formulated for every motion. Furthermore, since there is a phase difference between the arm and hand in the flapping and feathering motions (see Fig. 5), individual angle functions were formulated for the arm and hand. The angle function of the arm was defined on the basis of the body while the angle function of the hand was defined on the basis of the arm (see Fig. 6).

\subsection{Method to Calculate Wingbeat Force}

When a fixed wing is placed in an air flow expressed by the vector $v_{r}$, a lift force $\mathrm{L}$ and drag force $\mathrm{D}$ act on that wing. The force acting on the wing is determined as the resultant of these forces (see Fig. 7). L and D are expressed by the following formulae:

$$
\begin{aligned}
& L=\frac{1}{2} \cdot \rho \cdot v_{r}^{2} \cdot S \cdot C_{l}(\alpha) \\
& D=\frac{1}{2} \cdot \rho \cdot v_{r}^{2} \cdot S \cdot C_{d}(\alpha)
\end{aligned}
$$

where

$\rho$ : density of air $\left(\mathrm{g} / \mathrm{cm}^{3}\right)$,

$S$ : wing area $\left(\mathrm{cm}^{2}\right)$,

$\alpha$ : attack angle of wing elevation (deg),

$v_{r}$ : velocity of the air flow relative to the wing $\left|\boldsymbol{v}_{\boldsymbol{r}}\right|$,

$\mathrm{C}_{l}$ : lift coefficient expressed as a function of $\alpha$,

$\mathrm{C}_{d}$ : drag coefficient expressed as a function of $\alpha$.

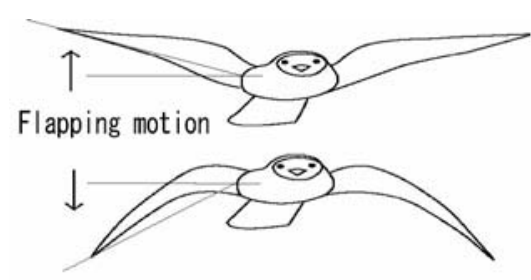

(a) Flapping

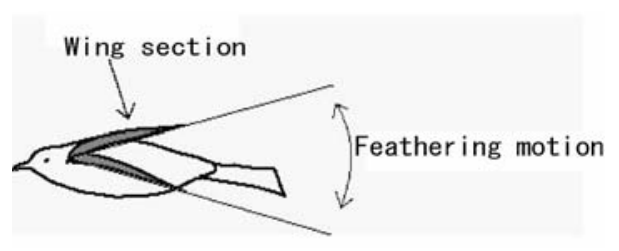

(b) Feathering

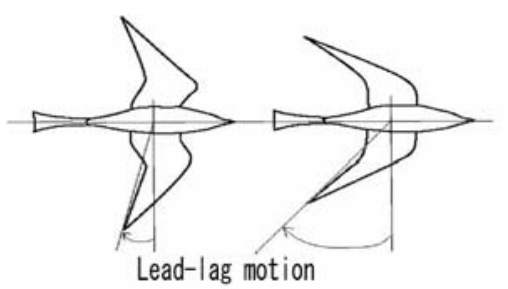

(c) Lead-lag

Fig. 2 Action of bird wings 


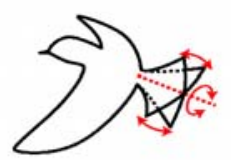

(a) Right/left twisting of tail feathers

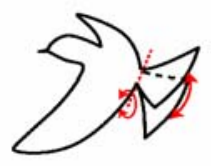

(b) Up/down bending of tail feathers

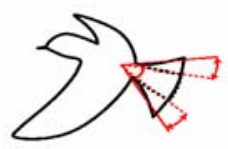

(c) Opening-and-closing angle of tail feathers

Fig. 3 Action of bird tail feathers

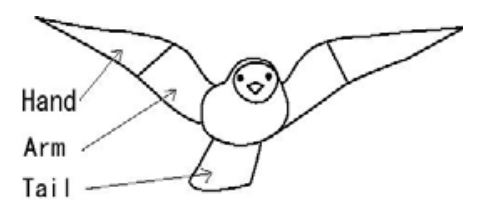

Fig. 4 Bird model

The attack angle of wing elevation $\alpha$ is the angle between the vector $v_{r}$ and tangent to the wing. The functions shown in Fig. 8 are used to calculate $C_{l}$ and $C_{d}$.

Further, the force acting on the entire wing must be calculated as the integral of the force at each point from the shoulder to the wing tip. In order to reduce the number of calculations required, two points $\mathrm{P}_{\text {arm }}$ and $\mathrm{P}_{\text {hand }}$ are assumed along the wing, one on the arm and the other on the hand. $\mathrm{P}_{\text {arm }}$, at which the average force on the arm is obtained, and $\mathrm{P}_{\text {hand }}$, at which the average force on the hand is obtained, are used as point forces to calculate velocity vectors and the flapping force (see Fig. 9).
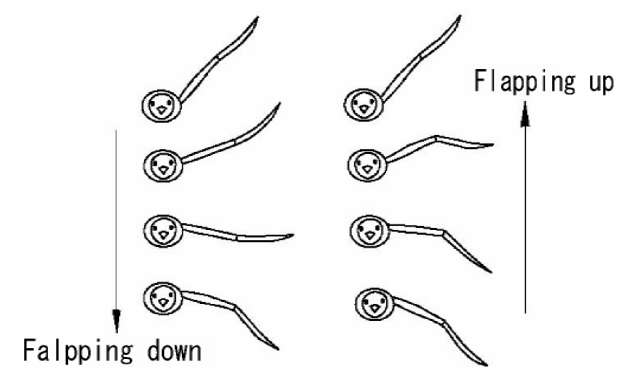

Fig. 5 Phase difference between arm and hand

\subsection{Method of Controlling Movement with Target Points}

The method of controlling the flight path involves placing arbitrary target points along the flight path and adjusting the left/right and up/down circling motions with respect to the direction of forward motion and bird's height such that the bird passes through the target points in sequence. The control points are considered to be spheres with finite radii, and a bird is considered to pass through a target point when it enters the sphere.

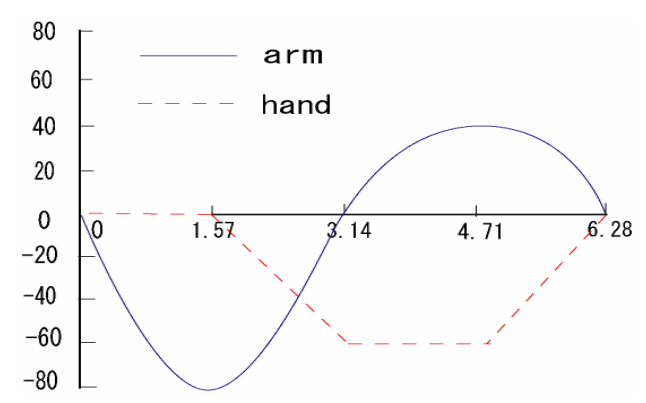

(a) Angle function of flapping motion

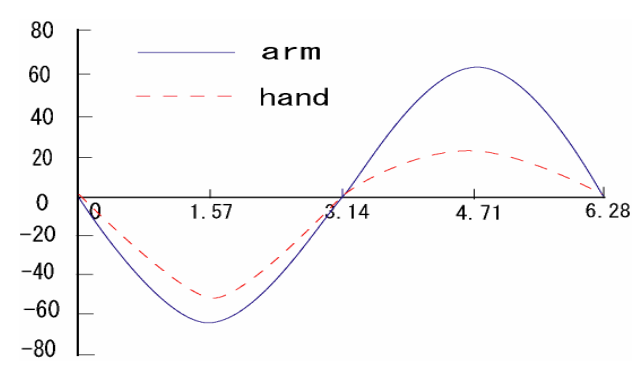

(b) Angle function of feathering motion

Vertical axis: Movement angle [deg], Horizontal axis: Phase [rad]

Fig. 6 Angle function of wingbeat

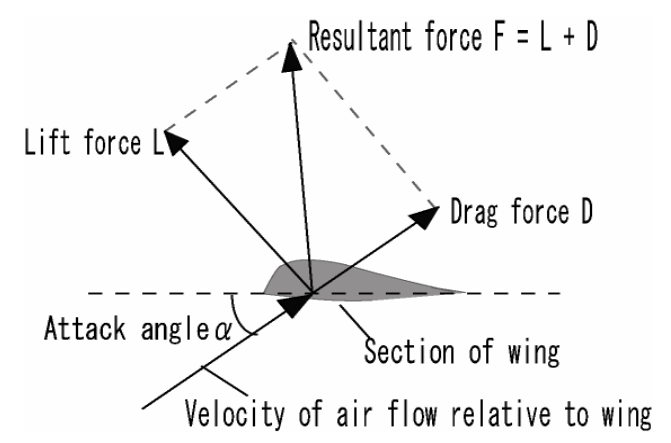

Fig. 7 Force acting on the wing

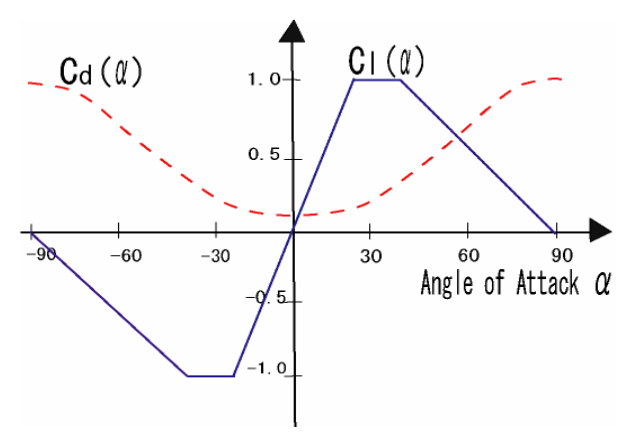

Fig. $8 \mathrm{C}_{l}$ and $\mathrm{C}_{d}$ functions 


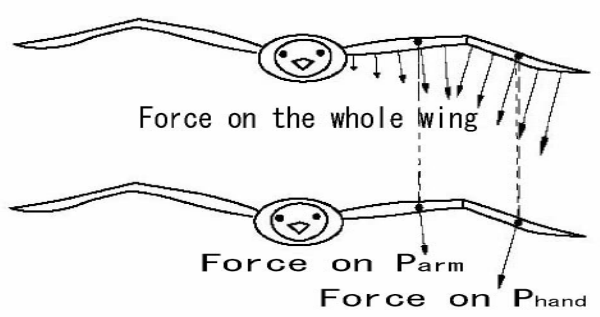

Fig. 9 Approximation with points $\mathrm{P}_{\text {arm }}$ and $\mathrm{P}_{\text {hand }}$

The bird's local coordinate system and rotational movement are shown in Fig. 10. Three types of rotational movement are defined: rolling (the $\mathrm{x}$-axis is the axis of rotation), yawing (the $y$-axis is the axis of rotation), and pitching (the z-axis is the axis of rotation). In the circling motion, the left/right rotation (rotation around the y-axis) and up/down rotation (rotation around the z-axis) are performed at the same time around the bird's center of gravity such that the angle between the horizontal component of the direction vector that expresses the direction of the bird's head and the horizontal component of the vector from the bird to the target point becomes zero. Further, in order to avoid the skid that accompanies rotation, a banking motion is simultaneously carried out along with the rotational movement (see Fig. 11). The rotation velocity $\omega$ is calculated by the following formula.

$$
\omega=-\operatorname{sgn}(\phi) R
$$

where

$R$ : standard rotation velocity,

$\phi$ :angle shown in Fig.11,

sgn: +1 while $\phi>0$, 0 while $\phi=0,-1$ while $\phi<0$.

The height is adjusted by controlling the flapping angle, feathering angle, and frequency.

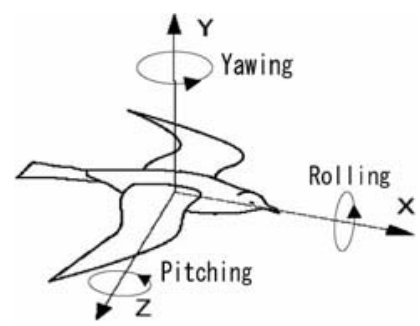

Fig. 10 Bird's local coordinate system and rotation

When the altitude of a bird is lower than a target point, it is adjusted by increasing wingbeat frequency and amplitude, and when it is higher than a target point, reducing wingbeat frequency and amplitude will be done. The wingbeat frequency is adjusted by the following formula.

$$
f(t+\Delta t)= \begin{cases}f_{\max } & f_{\max }<f(t) \\ f(t)+\operatorname{sgn}\left(y_{p}-y_{i}\right) K_{f} \Delta t & f_{\text {min }} \leq f(t) \leq f_{\max } \\ f_{\min } & f(t)<f_{\min }\end{cases}
$$

where $f_{\max }$ and $f_{\min }$ are maximum frequency and minimum frequency. $K_{f}$ is the amount of frequency displacement in one second, $y_{p}$ and $y_{i}$ are $y$ coordination values of the target point and the bird respectively. Wingbeat amplitude is adjusted by the following formula.

$a m p(t+\Delta t)= \begin{cases}a m p_{\max } & a m p_{\max }<a m p(t) \\ a m p(t)+\operatorname{sgn}\left(y_{p}-y_{i}\right) K_{a} \Delta t & a m p_{\text {min }} \leq a m p(t) \leq a m p_{\max } \\ a m p_{\text {min }} & a m p(t)<a m p_{\text {min }}\end{cases}$

where $a m p_{\max }$ and $a m p_{\min }$ are the maximum amplitude and minimum amplitude. $K_{\alpha}$ is the amount of amplitude displacement in one second, $y_{p}$ and $y_{i}$ are y coordination values of the target point and the bird respectively.

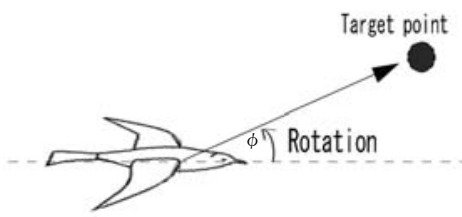

(a) Up/down rotation

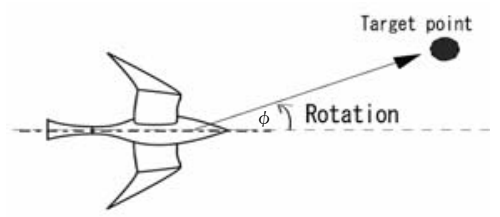

(b) Left/right rotation

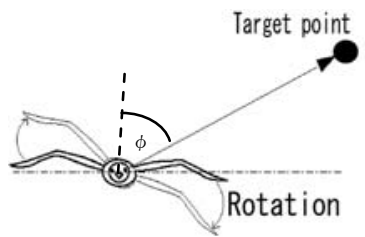

(c) Banking movement

Fig. 11 Controlling the direction of a bird at a target point

\subsection{Equation of motion for a bird}

The equation of motion for a bird is expressed as follows.

$$
f(t)=m \cdot a(t)
$$

where,

$f(\mathrm{t})$ and $a(\mathrm{t})$ are vector force and vector acceleration acting on the bird at time $t, m$ is the mass of the bird. The position $p(\mathrm{t})$ and velocity $v(\mathrm{t})$ are calculated by using the following finite difference equation derived by the Euler method.

$$
\begin{aligned}
& v(t+\Delta t)=v(t)+a(t) \cdot \Delta t \\
& p(t+\Delta t)=p(t)+v(t) \cdot \Delta t
\end{aligned}
$$

\section{Extension of Bird Flight Model}

The extension method of the bird flight model is described in this section.

\subsection{Controlling Direction of Bird by Tail Feathers}

As explained in section 2, the tail feathers are important for changing the advance direction and compensating for the climbing force. In this method, three flexible motions, namely, the twist to the left/right direction, bending in the up/down direction, and the opening-and-closing 
angle(See Fig.3) are pertinent to the tail feathers, and the direction and the amount of the force acting on the tail feathers are controlled by these three motions. When a target point is above the altitude of a bird, tail feathers are bent upward, and when a target point is under the bird, tail feathers are bent downward. The bent angle is set to pbend. Moreover, when a target point is in the right-hand side of a bird, the tail feathers is twisted to the left, and the motion of twisting to right is done when a target point is in left-hand side. The rotation angle is set to prot (see Fig.12).

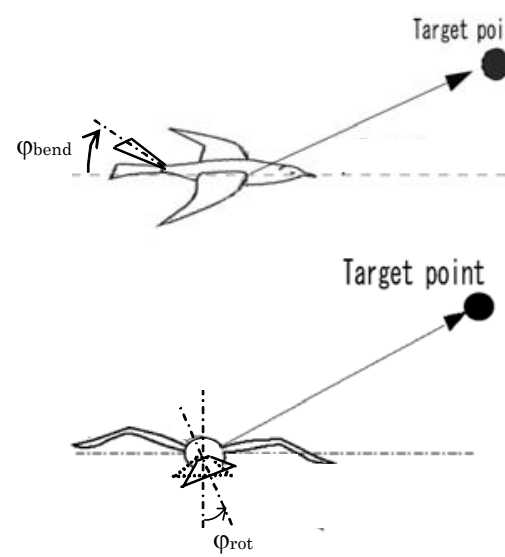

Fig.12 Control of tail feathers

The tail is approximated to a fan-like form and its area St is calculated from the opening-and-closing angle $\varphi$.

$$
S t=\frac{\pi l r^{2} \varphi}{360}
$$

where,

Ir: length of tail feathers.

$\varphi$ is calculated by the following formula.

$$
\varphi=C t\left|\max \left(\varphi_{\text {bend }}, \varphi_{\text {rot }}\right)\right|+\varphi_{\text {min }}
$$

where, $C t$ is a constant, $\varphi_{\min }$ is a minimum angle.

The force $\left(F_{t}\right)$ acting on the tail feathers is calculated to be the same as that acting on the wings.

The manner in which the force $F_{t}$ acting on the tail feathers should be used in order to control the bird flight direction is explained as follows. First, the body of the bird is approximated by a rectangular parallelepiped, and the length of the edges of this parallelepiped are set to a, b, and c (see Fig.13). Next, by assuming the rectangular parallelepiped to be a rigid body with a uniform density, the rotational movement equation about $\mathrm{F}_{\mathrm{t}}(\mathrm{t})$ is solved. This equation is expressed by the following formula.

$$
I \boldsymbol{\beta}(t)=\boldsymbol{r} \times \boldsymbol{F}_{t}(t)
$$

where

$I$ : inertia moment,

$\beta(\mathrm{t})$ : angular acceleration,

$r$ : length between the rotation center and point of action,

$\times$ : outer product of vector,

$F_{t}(t)$ : force vector at the point of action.

Additionally, the inertia moment $I$, acting on the center of gravity of the rectangular parallelepiped is expressed as follows:
$I_{x}=m \frac{b^{2}+c^{2}}{12}, I_{y}=m \frac{c^{2}+a^{2}}{12}, I_{z}=m \frac{a^{2}+b^{2}}{12}$

where

$m$ : mass of the bird,

$I_{x}, I_{y}, I_{z}$ : inertia moment of the circumference of each axis of the rectangular parallelepiped.

By using formulae 11 and 12, we calculate the angular acceleration on the rotation of the circumference of each axis of a bird. By using formulae 13 and 14 derived using the Euler method, the angular velocity $\omega(t)$ and rotation angle $\theta(t)$ are calculated as follows.

$$
\begin{aligned}
& \omega(t+\Delta t)=\omega(t)+\boldsymbol{\beta}(t) \cdot \Delta t \\
& \boldsymbol{\theta}(t+\Delta t)=\boldsymbol{\theta}(t)+\boldsymbol{\omega}(t) \cdot \Delta t
\end{aligned}
$$

Here, $\beta(\mathrm{t}), \omega(\mathrm{t})$, and $\theta(\mathrm{t})$ are expressed as vectors, their directions are expressed in the vector directions, and their magnitudes are expressed by the angular acceleration or angular velocity.

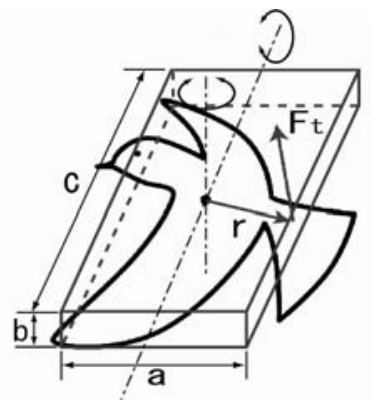

Fig. 13 Approximation of body for rotation

\subsection{Ground Effect}

The ground effect is a phenomenon in which the lift force increases when a bird flies close to the surface of the ground[8]. Since the magnitude of the ground effect depends on the height of the bird from the ground, the force $F_{\text {ground }}$ in the direction opposite to gravity is defined by the following formula, which is a function of the distance between the ground and bird.

$$
F_{\text {ground }}(h)=\left\{\begin{array}{cc}
k \frac{-G \cdot m}{h} & (0<h / l<1) \\
0 & \text { (others) }
\end{array}\right.
$$

where

$G$ : gravity acceleration,

$m$ : mass of the bird,

$h$ : height,

l: wing span,

$k$ : adjustment coefficient.

By balancing this force with the gravity when the bird lands, the bird can be prevented from diving into the ground.

\subsection{Method of Flying Away from Ground}

When a bird flies away from the ground, it can acquire an initial flight velocity from the takeoff. At this instant, the bird acts in a manner similar to that of person jumping, and it lowers its body for a brief duration to conserve power for the takeoff [8]. In this model, the force produced by its legs during takeoff while flying away from the ground is assumed in advance and a smooth 
simulation of the motion can thus be produced.

\subsection{Method of Controlling Flight Path for Landing on Branch}

In order to land on a branch, the bird has to advance perpendicularly toward the direction in which the branch is extended, as viewed from over the flight path of the bird. For this reason, a temporary target point is set at position $\mathrm{P}$ shown in Fig.14, and the bird flight is controlled so that the bird can advance perpendicularly toward a branch in this model. Here, P is defined as the center of the Bézier curve that has target points Q1, Q2, and Q3. Q1 and Q2 are the position of the bird and branch, respectively, and Q3 is the intersection of the line extended from Q1 in the direction of advance of the bird and the line extended from Q2 perpendicular to the branch.

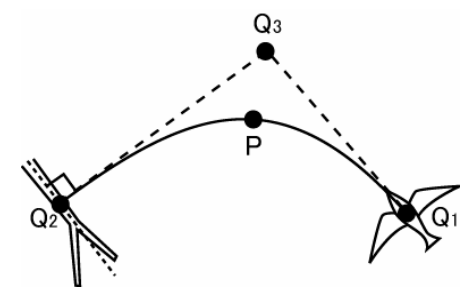

Fig. 14 Controlling flight path for landing on a branch

\section{Simulation Results}

The computer for simulations is DELL Inspiron1150 (CPU Celeron 2.4GHz, RAM 256MB, VRAM 64MB). The sampling interval of the Euler method is 0.0003 second, and rendering was performed at rate of one time in 100 times of calculations. The frame rate is about 40 fps.

The parameters used in simulations are shown in Table 1. Fig.15 expressed an actual bird of circular flight, captured from the video of birds [10]. Fig.16 is an example of the simulation which performed circular flight same as the bird flight of Fig.15. It turns out to be that the tail feathers of the bird in our simulation are acting like that of the actual bird. The movie file of simulation is Fig16.mpg.

A simulation of the ground effect is shown in Fig.17. A bird may be hidden under the ground when there is no ground effect; however, when the ground effect is taken into consideration, the bird does not dive into the ground.

Fig.18 shows a simulation of the flight path control for landing on a branch. In the case of no flight path control, a bird flies toward a branch directly, while it advances perpendicular to a branch when using the method of flight path control.

A simulation of flying away from the ground is shown in Fig.19 and Fig.20. Fig.19 shows the bird flight when there is no force produced by the takeoff, and Fig.20 is the case that considers the force produced by the takeoff. When considering the force produced by takeoff, a bird acquires initial velocity and its ascent is quicker. In this simulation, it is assumed that the takeoff force produced by legs is $2.1 \times 10^{6} \mathrm{dyn}$ and its time acting on the legs will take 0.1 second. The movie file is Fig19-20.mpg.
Shown in Fig.21, it is an example of moving target points while simulating. It turns out that a flight course can be dynamically changed by moving target points while performing a flight. The result shows that when our technique is implemented in a driving simulator, etc, it is possible to make the bird perform action like avoiding moving cars. The movie file is Fig21.mpg.

\section{Conclusion}

This paper proposed an extension method of Cao's bird flight model by which a bird can fly in real time. In this method, the motions produced by tail feathers were taken into consideration in the rotational movement. Furthermore, methods were proposed to consider takeoff while the bird flies away, the ground effect, dynamic target points change flight and the process of landing on a branch. The effect of these methods was demonstrated by simulations. The inclusion of hovering flight and foldable wings will be the subject of future research.

\section{References}

[1] C. W. Reynolds: Flocks, herds and schools, A distributed behavioral model, SIGGRAPH '87, pp.25-34 (1987).

[2] B. Ramakrishnananda, K. C. Wong: Animating bird flight using aerodynamics, The Visual Computer, No. 10, pp. 494-508 (1999).

[3] J. Wu, Z. Popovic: Realistic modeling of bird flight animations, SIGGRAPH 2003, pp. 888-895 (2003).

[4] The pictorial book of birds (in Japanese), Syougakukan (1988).

[5] M. Handa: Techno Life selected book Flight (structure and fluid dynamics) (in Japanese), Ohmsha (1994).

[6] C. Cao, T. Fujimoto, K. Muraoka, and N. Chiba: Visual simulation of bird flight (in Japanese), NICOGRAPH /MULTIMEDIA, No. 17, pp. 93-98 (2001).

[7] Y. Anzai, K. Muraoka, N. Chiba and N. Saito: A flight model for butterflies taking into account the force caused by flapping the wings (in Japanese), Transaction of the Information Processing Society of Japan, Vol. 41, pp. 658-667 (1999).

[8] A. Azuma: Creature, its wonderful motion (in Japanese), Kyoritu Publishing (1986).

[9] C. Zhu, K. Muraoka, T. Kawabata, C. Cao, T. Fujimoto, N. Chiba: Real-time Animation of Bird Flight Based on Aerodynamics, Proceedings of NICOGRAPH International 2005, PP. 117-122, 2005.

[10]D. Attenborough: The Life of Birds, Vol.4, BBC Worldwide Ltd, 1998 
芸術科学会論文誌 Vol.5 No.1 pp.1-10
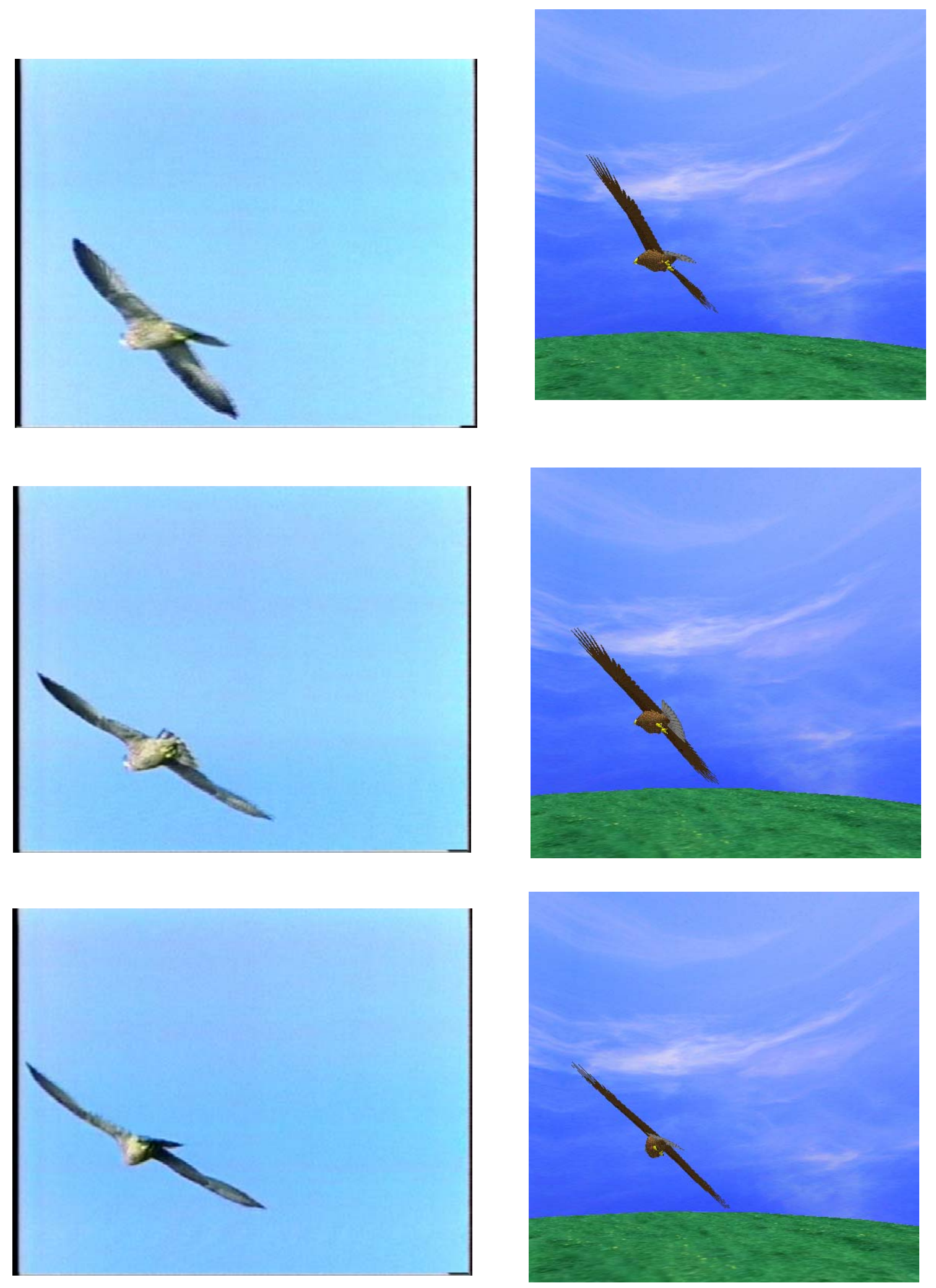

Fig. 15 Circular flight by real bird [10]

Fig.16 Circular flight by using tail feathers 
芸術科学会論文誌 Vol.5 No.1 pp.1-10

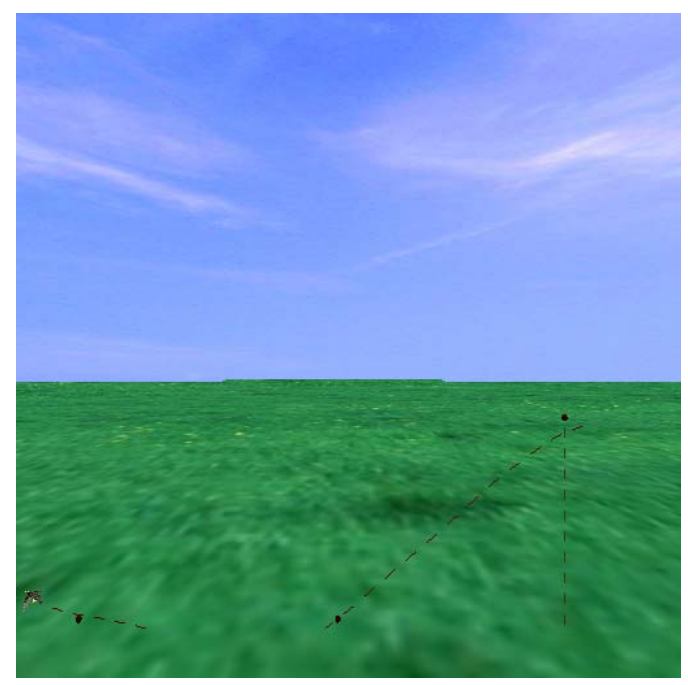

(a) No ground effect

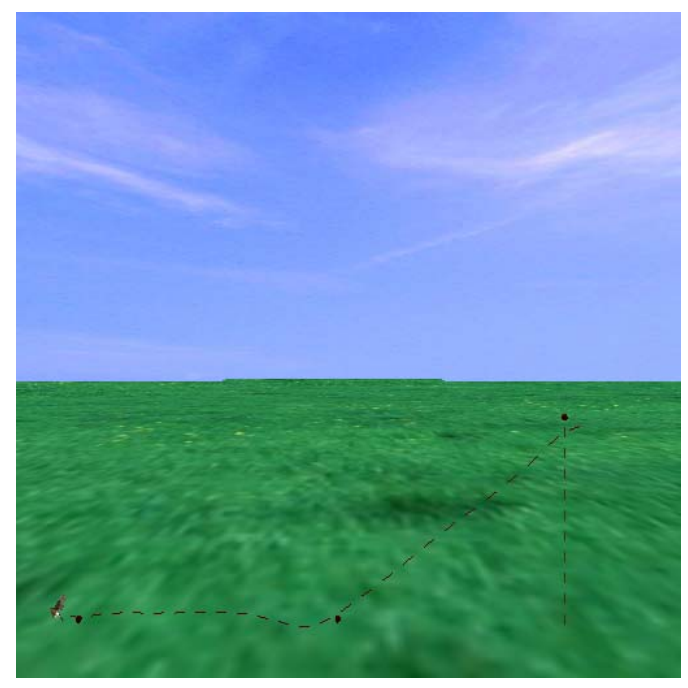

(b) Ground effect

Fig. 17 Simulation of ground effect

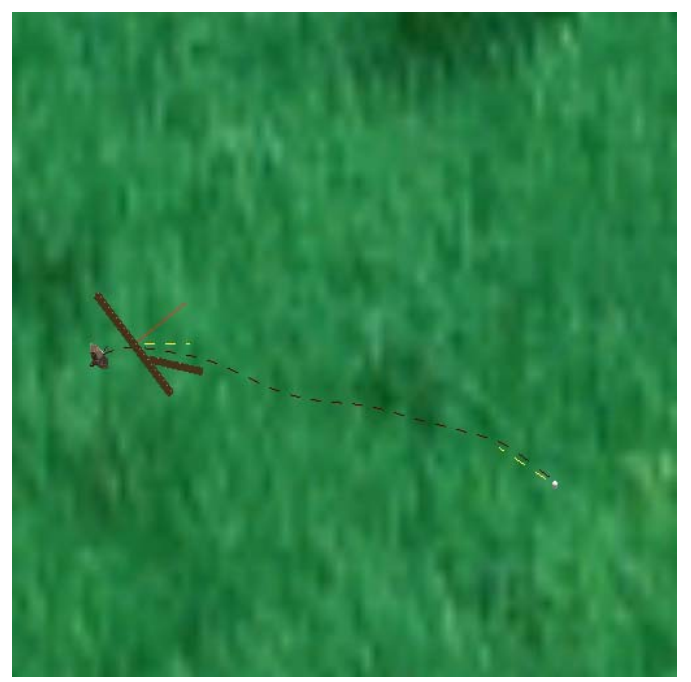

(a) Conventional method

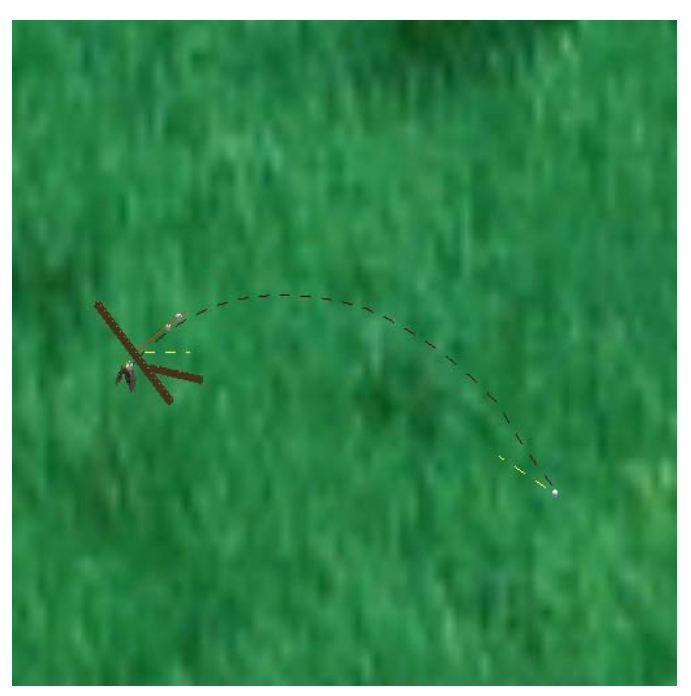

(b) Method with flight path control

Fig. 18 Control to stop at a branch 
芸術科学会論文誌 Vol.5 No.1 pp.1-10

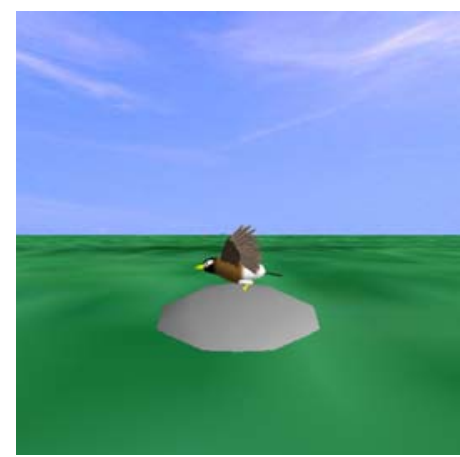

(a) 0.0 second

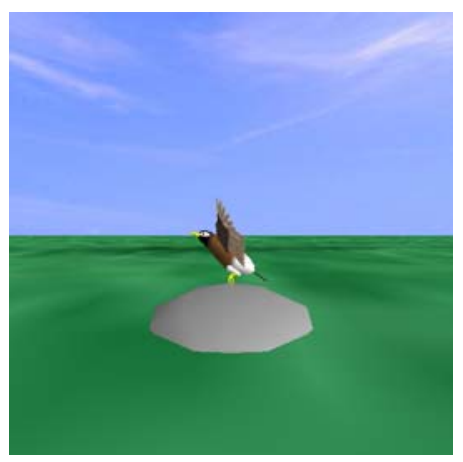

(b) 0.15 second

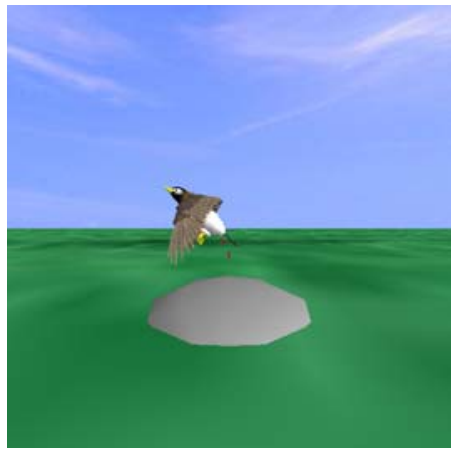

(c) 0.3 second

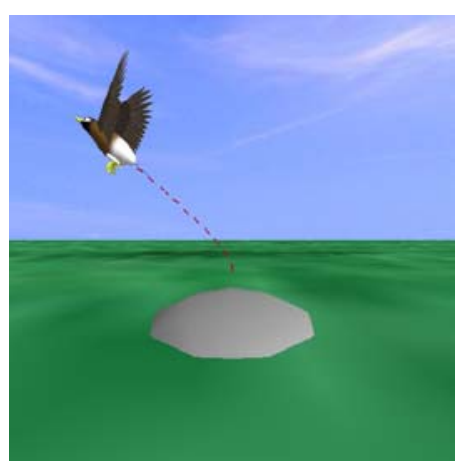

(d) 0.7 second

Fig. 19 Flying away with no force of takeoff

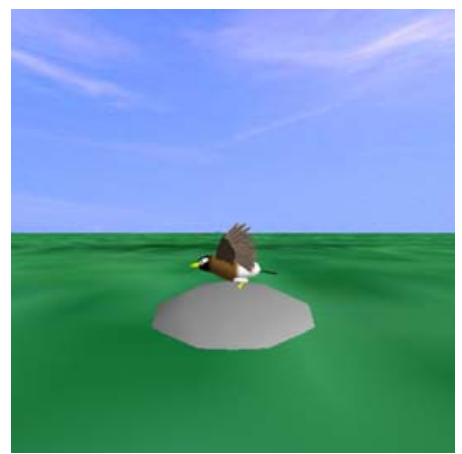

(a) 0.0 second

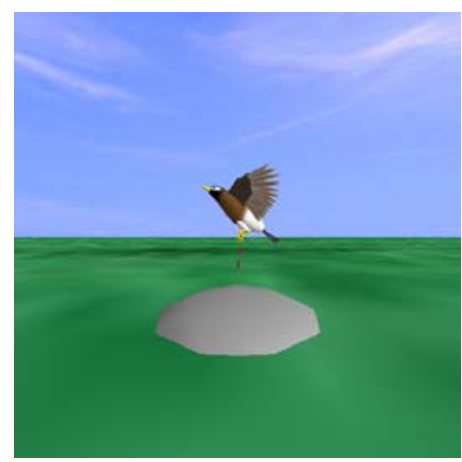

(b) 0.15 second

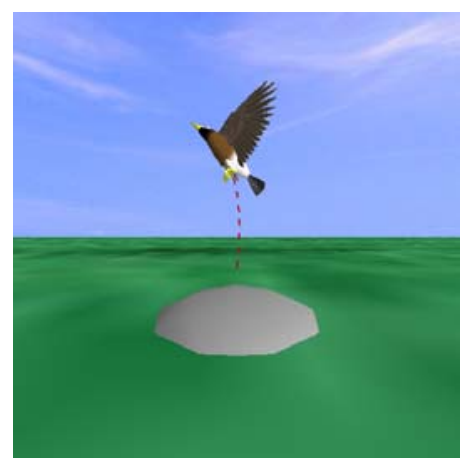

(c) 0.3 second

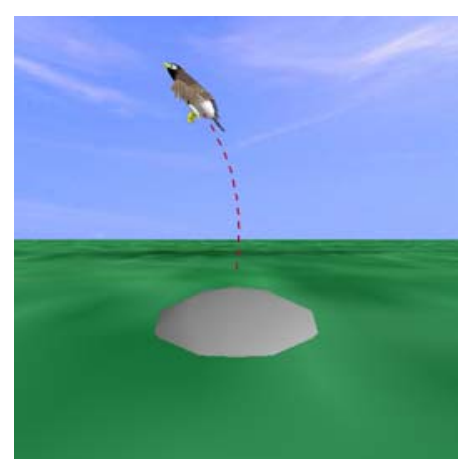

(d) 0.7 second

Fig. 20 Flying away with force of takeoff 
芸術科学会論文誌 Vol.5 No.1 pp.1-10

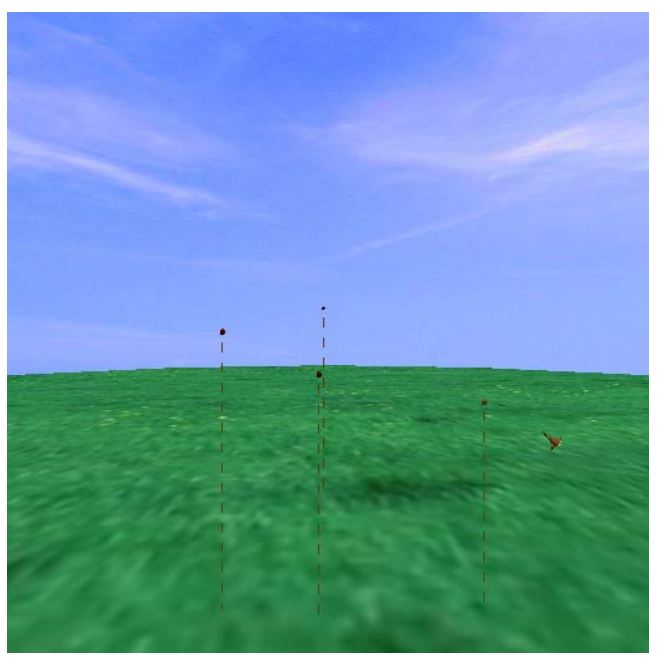

Table 1 Simulation parameters

\begin{tabular}{|c|c|c|}
\hline Parameter & $\begin{array}{c}\text { Fig. 16 } \\
\text { (Eagle) }\end{array}$ & $\begin{array}{c}\text { Fig.17 21 } \\
\text { (Starling) }\end{array}$ \\
\hline $\mathrm{S}\left(\mathrm{cm}^{2}\right)$ & 7200 & 1760 \\
\hline$m(\mathrm{~g})$ & 2000 & 500 \\
\hline$f_{\max }, f_{\min }(\mathrm{Hz})$ & 5,2 & 10,8 \\
\hline $\mathrm{K}_{f}$ & 0.36 & 1.0 \\
\hline$a m p_{\max }, a m p_{\min }$ & $1.0,0.0$ & $1.0,0.6$ \\
\hline $\mathrm{K}_{a}$ & 0.25 & 0.25 \\
\hline $\mathrm{a}, \mathrm{b}, \mathrm{c}(\mathrm{cm})$ & $80,20,220$ & $40,10,100$ \\
\hline$I r(\mathrm{~cm})$ & 40 & 20 \\
\hline$\varphi_{\text {bend }}\left({ }^{\circ}\right)$ & \pm 10 & \pm 10 \\
\hline$\varphi_{\text {rot }}\left(^{\circ}\right)$ & \pm 45 & \pm 60 \\
\hline$\varphi_{\min }\left({ }^{\circ}\right)$ & 10 & 10 \\
\hline $\mathrm{Ct}$ & 1.78 & 1.78 \\
\hline $\mathrm{k}$ & 10 & 10 \\
\hline$l(\mathrm{~cm})$ & 90 & 40 \\
\hline
\end{tabular}
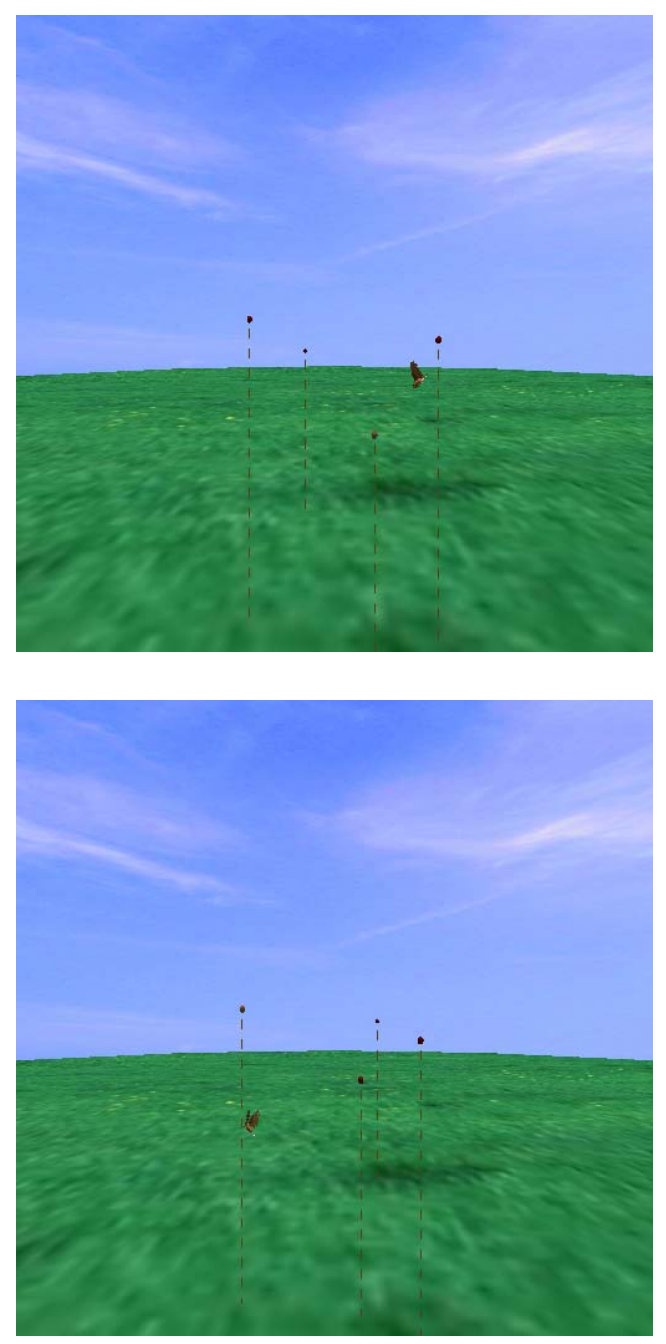

Fig.21 Simulation of moving target points 